Artículo de investigación

\title{
DISTRIBUCIÓN ESPACIAL, ESTRUCTURA Y VOLUMEN DE LOS BOSQUES DE ROBLE NEGRO (Colombobalanus excelsa (Lozano, Hern. Cam. \& Henao, J.E.) Nixon \& Crepet) EN EL PARQUE NACIONAL NATURAL CUEVA DE LOS GUÁCHAROS
}

\section{Spatial distribution structure and volume of Colombian black oak forest (Colombobalanus excelsa (Lozano, Hern. Cam. \& Henao, J.E.) Nixon \& Crepet) National Natural Park Cueva de los Guácharos}

Palabras clave: bosque, distribución, forma, modelo, roble, volumen.

Key words: forest, distribution, shape, model, oak, volume.
David Eduardo Dávila'

José Franco Alvis ${ }^{2}$

Román Ospina

\section{RESUMEN}

Se determinó la distribución espacial de los bosques dominados Colombobalanus excelsa en el Parque Nacional Natural (PNN) Cueva de los Guácharos y su zona de amortiguación y se precisaron sus parámetros estructurales utilizando un inventario forestal estratificado. El inventario se realizó en cuatro fajas de 0.5 ha distribuidas en dos estratos: el primer estrato se localizó en el PNN Cueva de los Guácharos y el segundo en su zona de amortiguación. Cada faja estuvo conformada por parcelas de $20 \times 50 \mathrm{~m}$ para registrar individuos con diámetros a la altura del pecho (DAP) $\geq 10 \mathrm{~cm}$, altura total, diámetro de copa y estado del árbol; asimismo, en cada faja se anidó una subparcela de $10 \times 10 \mathrm{~m}$ para registrar individuos con $\mathrm{DAP} \leq 10 \mathrm{~cm}$ y alturas mayores a $3 \mathrm{~m}$ y el número de plántulas $\geq 0.3 \mathrm{~m}$ de altura se estimó en subparcelas de $5 \mathrm{x}$ $5 \mathrm{~m}$. Se generaron modelos para estimar la altura $y$ el volumen en función del DAP. Se reportaron un total de ocho rodales naturales de roble negro que alcanzaban las 2000 ha, de las cuales 28.3 ha se encontraron dentro del parque. Se registró una densidad de 281.7 árbolesha $^{-1}$, un área basal de $52.33 \mathrm{~m}^{2}$ ha $^{-1}$ y un volumen de $761.65 \mathrm{~m}^{3} \mathrm{ha}^{-1}$. El factor mórfico para la especie fue de 0.76041 . Se ajustaron seis modelos para estimar la altura y seis para volumen con ajustes de 0.90 y 0.98 , respectivamente.

\section{ABSTRACT}

The spatial distribution of Colombobalanus excelsa forests in the Cueva de los Guácharos Natural National Park and its buffer zone was determined. The forest's structural parameters were determined by conducting a stratified forest inventory that consisted of four plots of 0.5 ha distributed in two strata. The first stratum was located in the park and the second in its buffer zone. Each strip consisted of plots of $20 \times 50 \mathrm{~m}$ within which individuals with diameters at breast height $\geq 10 \mathrm{~cm} \mathrm{DBH}$ were measured for total height, crown diameter and the condition of each tree. Within each strip a $10 \times 10 \mathrm{~m}$ subplot was used to assess individuals with DBH $\leq$ $10 \mathrm{~cm}$ and heights greater than $3 \mathrm{~m}$. In addition the number of seedlings of height $\geq 0.3 \mathrm{~m}$ were counted in subplots of $5 \times 5 \mathrm{~m}$. Models were generated to estimate the height and volume as a function of $\mathrm{DBH}$. We report a total of eight natural stands of black oak reaching 2000 ha of which 28.3 ha were found within the park. We report a density of 281.7 trees ha ${ }^{-1}$ with a basal area of $52.33 \mathrm{~m}^{2} \mathrm{ha}^{-1}$ and a volume of $761.65 \mathrm{~m}^{3} \mathrm{ha}^{-1}$. The form-factor for the species was of 0.76041 . Six models were fitted to estimate the height and six for volume adjustments of 0.90 and 0.988 , respectively.

\footnotetext{
Universidad del Cauca. Popayán, Colombia. leonn329@hotmail.com

Universidad del Cauca. Popayán, Colombia. falvis@unicauca.edu.co

Universidad del Cauca. Popayán, Colombia.rospina@unicauca.edu.co. Autor para correspondencia.
} 


\section{INTRODUCCIÓN}

El roble negro (Colombobalanus excelsa) es una especie que forma asociaciones con altas densidades de individuos, en zonas con fuertes pendientes y suelos pobres; por lo general, en terrenos adyacentes a bosques de roble blanco (Quercus humboldtii) (IAVH, 2002; Manchola \& Parra, 2006). De acuerdo con Cárdenas \& Salinas (2006), C. excelsa (Fagaceae) es una especie endémica de los Andes colombianos considerada vulnerable y con una extensión cercana a los 1100 $\mathrm{km}^{2}$, cuyas poblaciones solo se han registrado en los parques nacionales naturales Cueva de Los Guácharos (Huila), Farallones de Cali (Valle del Cauca) y en el Santuario de Flora y Fauna Guanentá alto Río Fonce (Santander). Los registros señalan que la especie ocurre entre los 1800 y $2200 \mathrm{~m}$ de altitud, con una temperatura media anual de $15.4^{\circ} \mathrm{C}$ y una precipitación promedio de $2996 \mathrm{~mm}$ al año. Generalmente, crecen en condiciones de bosque nublado y luminoso en los flancos internos de las cordilleras de los andes colombianos (Fundación Farallones, 2009).

En el departamento del Huila se ha registrado esta especie desde los $1400 \mathrm{~m}$ de altitud, donde no alcanza a formar rodales monoespecíficos (Manchola \& Parra, 2006). Las plántulas y árboles juveniles son heliófilos, sus frutos son consumidos por ardillas y aves quienes dispersan las semillas, mientras que la polinización se realiza a través del viento (Fundación Farallones, 2009). Dávila (2010) estimó que el área aproximada cubierta por rodales de C. excelsa, en la zona de amortiguación del PNN Cueva de los Guácharos, es de 2000 ha. En esta zona se practican actividades de aprovechamiento de la especie sin orientación técnica, lo que hace necesario conocer mejor aspectos ecológicos de estos bosques, así como también parámetros productivos con el fin de poder orientar la actividad forestal hacia sistemas de producción sostenible de largo plazo como lo sugieren Wadsworth (1997) y Louman et al. (1991). Además, algunos rodales presentes en la zona de amortiguación del parque están constituidos por fragmentos, esto puede implicar que están sometidos a efectos relacionados con la fragmentación como cambios de temperatura, vientos y radiación, entre otros (Williams, 1991; Bustamante \& Grez, 1995; Murcia, 1995; Kattan, 2002). En este sentido, el presente trabajo tuvo como propósito generar algunas bases técnicas para ayudar a facilitar futuras investigaciones con la especie $C$. excelsa, así como para orientar las prácticas de manejo forestal en los bosques dominados por esta. Un primer objetivo consistió en conocer la distribución espacial de los bosques de roble negro en el PNN Cueva de los Guácharos y su zona de amortiguación; en segundo lugar, determinar sus características ecológicas y silviculturales y por último, determinar el factor mórfico para la especie y se ajustar modelos para estimar la altura y volumen de los árboles en función del diámetro a la altura del pecho.

\section{MATERIALES Y MÉTODOS}

El trabajo se realizó en el Parque Nacional Natural Cueva de los Guácharos y su zona de amortiguación, entre las coordenadas $1^{\circ} 37^{\prime} 56^{\prime \prime} \mathrm{N}, 76^{\circ} 06^{\prime} 10^{\prime \prime}$ W y $1^{\circ} 42^{\prime} 44^{\prime \prime} \mathrm{N}, 76^{\circ} 01^{\prime} 33^{\prime \prime} \mathrm{W}$, entre los 1500 y $2200 \mathrm{~m}$ de altitud. Para estudiar la distribución espacial de los bosques dominados por la especie C. excelsa, se tomó como base un mapa digital suministrado por la administración del parque. El mapa fue actualizado con información registrada en recorridos de campo tomando puntos y levantando polígonos correspondientes a los rodales, con ayuda de un GPS y con el empleo del sistema de coordenadas de Gauss con origen Occidente $4^{\circ} 35^{\prime} 56.57^{\prime \prime} \mathrm{N} ; 4^{\circ} 04^{\prime} 51.30^{\prime \prime} \mathrm{W}$ como base. A partir del mapa de distribución espacial actualizado se identificó la existencia de varios fragmentos de bosques de roble negro con intervención antrópica, al interior de la zona de amortiguación del parque, y solamente un fragmento sin intervención dentro del área de reserva. Considerando esta situación, se hizo un inventario forestal estratificado con dos estratos: uno en la zona de reserva y otro en la zona de amortiguación. En cada estrato se levantaron fajas de $20 \times 250 \mathrm{~m}$, tres en la zona de amortiguación y una en el área del parque; cada faja se dividió en parcelas rectangulares de $20 \times 5 \mathrm{~m}$, en las que se registraron los individuos con diámetros a la altura del pecho DAP $\geq 10 \mathrm{~cm}$, altura, diámetro de copa, estado fitosanitario y coordenadas locales. 
Para registrar la regeneración establecida de C. excelsa (individuos con DAP $\leq 10$ y alturas mayores a $3 \mathrm{~m}$ ), en cada faja se anidó una parcela de $10 \times 10 \mathrm{~m}$ y para registrar plántulas con alturas mayores de $0.3 \mathrm{~m}$ se delimitó una subparcela de 5 x $5 \mathrm{~m}$. Para la obtención del factor de forma para C. excelsa, se midieron los diámetros y las alturas de 25 árboles con un relascopio, considerando 5 estratos definidos por clases diamétricas $(10 \leq$ $\mathrm{DAP} \leq 30 \mathrm{~cm} ; 30<\mathrm{DAP} \leq 50 \mathrm{~cm} ; 50<\mathrm{DAP} \leq$ $70 \mathrm{~cm} ; 70<\mathrm{DAP} \leq 90$ y DAP $>90 \mathrm{~cm})$. El método para hallar el factor de forma fue el denominado factor de forma verdadero de Hohenald, citado por Prodán et al. (1997); con los datos de los 25 árboles se procedió a calcular su respectivo volumen total y a establecer las relaciones entre las variables registradas.

Para facilitar la estimación de las alturas, así como del volumen en pie de $C$. excelsa, se ajustaron seis modelos mediante el uso de los programas Curvexpert Profesional ${ }_{\text {Versión 1.6, }}$, el cual puede de manera automática ajustar modelos usando tablas de datos con pares de variables XY, mediante técnica de regresión lineal y no lineal, interpolación o estrias (Hyams, 2010), y el estadístico SPSS ${ }_{\text {versión }}$ ${ }_{17}$, con el cual se pueden importar datos de casi cualquier tipo de archivo y utilizarlos para generar informes tabulares, gráficos y diagramas de distribuciones y tendencias, estadísticos descriptivos y análisis estadísticos complejos (IBM, 2010). Por medio de este último software se pudieron realizar pruebas de normalidad de los datos y homogeneidad de varianzas, así como análisis de varianza para ayudar a validar la calidad de los modelos.

\section{RESULTADOS}

De acuerdo con el estudio de la distribución espacial de los bosques de roble negro en el PNN Cueva de los Guácharos y su zona de amortiguación, fueron reportados ocho rodales de roble negro (C. excelsa), que suman un total de 2000 ha, de los cuales siete fueron encontrados en la zona de amortiguación del parque. El rodal más extenso con 1321.1 ha se localizó en la zona de amortiguación en el municipio de Acevedo (Huila), mientras que dentro del parque solo se encontró un rodal con 28.3 ha (Tabla 1$)$.

Tabla 1. Rodales de roble negro (Colombobalanus excelsa) registrados para el PNN Cueva de los Guácharos y su zona de amortiguación. Área total 2000 ha

\begin{tabular}{|c|c|c|c|c|}
\hline Rodal & $\begin{array}{l}\text { Área } \\
\text { (ha) }\end{array}$ & Coordenadas & $\begin{array}{l}\text { Altitud } \\
\text { (m) }\end{array}$ & Zona \\
\hline \multirow{2}{*}{1} & \multirow{2}{*}{234.38} & $1^{\circ} 39^{\prime} 18^{\prime \prime} \mathrm{N}$ & 1590 & \multirow{2}{*}{ Amortiguación } \\
\hline & & $76^{\circ} 05^{\prime} 25^{\prime \prime} \mathrm{W}$ & 2030 & \\
\hline \multirow{2}{*}{2} & \multirow{2}{*}{13.35} & $1^{\circ} 39^{\prime} 47^{\prime \prime} \mathrm{N}$ & 2036 & \multirow{2}{*}{ Amortiguación } \\
\hline & & $76^{\circ} 06^{\prime} 45^{\prime \prime} \mathrm{W}$ & 2135 & \\
\hline \multirow{2}{*}{3} & \multirow{2}{*}{291.35} & $1^{\circ} 39^{\prime} 21^{\prime \prime} \mathrm{N}$ & 1645 & \multirow{2}{*}{ Amortiguación } \\
\hline & & $76^{\circ} 05^{\prime} 51^{\prime \prime} \mathrm{W}$ & 1948 & \\
\hline \multirow{2}{*}{4} & \multirow{2}{*}{36.22} & $1^{\circ} 41^{\prime} 07^{\prime \prime} \mathrm{N}$ & 1828 & \multirow{2}{*}{ Amortiguación } \\
\hline & & $76^{\circ} 05^{\prime} 44^{\prime \prime} \mathrm{W}$ & 1972 & \\
\hline \multirow{2}{*}{5} & \multirow{2}{*}{1321.11} & $1^{\circ} 41^{\prime} 11^{\prime \prime} \mathrm{N}$ & 1408 & \multirow{2}{*}{ Amortiguación } \\
\hline & & $76^{\circ} 01$ ' $16^{\prime \prime} \mathrm{W}$ & 1978 & \\
\hline \multirow{2}{*}{6} & \multirow{2}{*}{72.03} & $1^{\circ} 39^{\prime} 30^{\prime \prime} \mathrm{N}$ & 1501 & \multirow{2}{*}{ Amortiguación } \\
\hline & & $76^{\circ} 01 ’ 39^{\prime \prime} \mathrm{W}$ & 1704 & \\
\hline \multirow{2}{*}{7} & \multirow{2}{*}{2.977} & $1^{\circ} 41^{\prime} 52^{\prime \prime} \mathrm{N}$ & 1571 & \multirow{2}{*}{ Amortiguación } \\
\hline & & $76^{\circ} 04^{\prime} 20^{\prime \prime} \mathrm{W}$ & 1604 & \\
\hline \multirow{2}{*}{8} & \multirow{2}{*}{28.33} & $1^{\circ} 37^{\prime} 56^{\prime \prime} \mathrm{N}$ & 1794 & \multirow{2}{*}{ Parque } \\
\hline & & $76^{\circ} 06^{\prime} 03^{\prime \prime} \mathrm{W}$ & 1975 & \\
\hline
\end{tabular}

Por otra parte, se encontró que la densidad de individuos adultos de $C$. excelsa con diámetros a la altura del pecho mayores a $10 \mathrm{~cm}$ fue de 281.7 árboles $\mathrm{ha}^{-1}$, mientras que el área basal fue de $52.34 \mathrm{~m}^{2} \mathrm{ha}^{-1}$ y el volumen de $761.65 \mathrm{~m}^{3} \mathrm{ha}^{-1}$; como se observa en la tabla 2 . El coeficiente de variación $(\mathrm{CV} \%)$ para estos tres parámetros es relativamente bajo, lo que indica una homogeneidad de los mismos; además se encontró que el error de muestreo para las tres variables señaladas fue de $9.33 \%, 8.89 \%$ y $7.72 \%$, respectivamente, lo cual corrobora la baja variabilidad de estos atributos (Tablas 3 y 4). La distribución de los individuos por clase de diámetro fue en forma de “ $\mathrm{j}$ ” invertida, con un alto número de individuos en las clases inferiores; sin embargo, se encontró que existe una buena proporción de individuos en las últimas tres clases (Tabla 5). En este sentido se observó que el área basal de $27 \mathrm{~m}^{2} \mathrm{ha}^{-1} \mathrm{y}$ el volumen de $423 \mathrm{~m}^{3} \mathrm{ha}^{-1}$ en estas clases fueron altos, respecto de valores publicados por otros autores para algunas especies del género Quercus en Costa Rica, donde 
se registran entre 391 y $707 \mathrm{~m}^{3} \mathrm{ha}^{-1}$ (Blaser \& Camacho, 1991; Orozco, 1991).

El coeficiente mórfico para $C$. excelsa fue de 0.76 con un coeficiente de variación del $14 \%$, para un error de muestreo $4.87 \%$, esto indica que este parámetro es estable para las diferentes clases de diámetros evaluados. Por otro lado, los coeficientes mórficos resultaron ser mayores para las clases diamétricas más altas, lo cual indica que los árboles de esta especie se asemejan más a un cilindro a medida que su diámetro aumenta: la tabla 2 relaciona los coeficientes mórficos por clases de diámetro reportados por el muestreo.

Tabla 2. Parámetros de la estructura del rodal para los bosques de roble negro (Colombobalanus excelsa) en el PNN Cueva los Guácharos y su zona de amortiguación. Coeficiente de variación (CV), estimación mínima confiable (EMC) y error de muestreo (EM\%)

\begin{tabular}{lrrr}
\hline \multirow{2}{*}{ Estadísticos } & \multicolumn{3}{c}{ Parámetros } \\
\cline { 2 - 4 } & Densidad (individuos $\left.\mathbf{~ h a}^{-1}\right)$ & Área basal $\left(\mathbf{m}^{\mathbf{2}} \mathbf{h a}^{-1}\right)$ & Volumen $\left(\mathbf{m}^{\mathbf{3}} \mathbf{h a}^{-1} \mathbf{)}\right.$ \\
\hline Promedio & 281.7 & 52.3 & 761.7 \\
Desviación & 5.9 & 2.8 & 13.2 \\
CV\% & 2.1 & 5.3 & 1.7 \\
Error estandar & 4.2 & 2.0 & 9.3 \\
EMC & 255.4 & 47.7 & 702.8 \\
EM\% & 9.33 & 8.89 & 7.72 \\
\hline
\end{tabular}

Tabla 3. Factor mórfico registrado para cinco clases diamétricas en roble negro (Colombobalanus excelsa), PNN Cueva de los Guácharos ( $\mathrm{N}=25$ árboles, cinco por cada clase diamétrica)

\begin{tabular}{lrrrrr}
\hline \multirow{2}{*}{ Parámetro } & \multicolumn{5}{c}{ Clase de DAP $(\mathbf{c m})$} \\
\cline { 2 - 6 } & $\mathbf{1 0}<\mathbf{D A P} \leq \mathbf{3 0}$ & $\mathbf{3 0}<\mathbf{D A P} \leq \mathbf{5 0}$ & $\mathbf{5 0}<\mathbf{D A P} \leq \mathbf{7 0}$ & $\mathbf{7 0}<\mathbf{D A P} \leq \mathbf{9 0}$ & $\mathbf{D A P}>\mathbf{9 0}$ \\
\hline Factor mórfico & 0.67 & 0.70 & 0.72 & 0.83 & 0.89 \\
Desviación estándar & 0.07 & 0.09 & 0.07 & 0.04 & 0.09 \\
Error estándar & 0.03 & 0.04 & 0.03 & 0.02 & 0.04 \\
Coeficiente de variación & 9.95 & 13.46 & 9.04 & 5.30 & 9.65 \\
Error de muestreo & 9.49 & 12.83 & 8.62 & 5.05 & 9.20 \\
\hline
\end{tabular}

Tabla 4. Parámetros de la estructura del rodal para los bosques de roble negro (Colombobalanus excelsa) en los dos estratos parque y zona de amortiguación 2011. Coeficiente de variación (CV), estimación mínima confiable (EMC) y error de muestreo (E\%)

\begin{tabular}{|c|c|c|c|c|c|c|}
\hline \multirow[b]{2}{*}{ Estadísticos } & \multicolumn{3}{|c|}{ Parque } & \multicolumn{3}{|c|}{ Z. amortiguación } \\
\hline & $\begin{array}{c}\text { Densidad } \\
\left(\text { individuos } \mathrm{ha}^{-1}\right)\end{array}$ & $\begin{array}{c}\text { Área basal } \\
\left(\mathbf{m}^{2} \mathbf{h a}^{-1}\right)\end{array}$ & $\begin{array}{l}\text { Volumen } \\
\left(\mathbf{m}^{3} \mathbf{h a}^{-1}\right)\end{array}$ & $\begin{array}{c}\text { Densidad } \\
\left(\text { individuos } \text { ha }^{-1}\right)\end{array}$ & $\begin{array}{c}\text { Área basal } \\
\left(\mathbf{m}^{2} \mathbf{h a}^{-1}\right)\end{array}$ & $\begin{array}{l}\text { Volumen } \\
\left(\mathbf{m}^{3} \mathbf{h a}^{-1}\right)\end{array}$ \\
\hline Promedio & 240.0 & 56.3 & 854.8 & 323.3 & 43.2 & 668.5 \\
\hline Desviación & 5.1 & 1.9 & 31.4 & 4.0 & 2.9 & 47.2 \\
\hline $\mathrm{CV} \%$ & 10.7 & 3.3 & 3.7 & 6.3 & 6.7 & 7.1 \\
\hline Error estándar & 2.3 & 0.8 & 14.0 & 2.3 & 0.7 & 12.2 \\
\hline EMC & 235.1 & 54.3 & 821.8 & 273.3 & 41.9 & 647.0 \\
\hline EM\% & 10.2 & 3.5 & 3.9 & 7.7 & 3.0 & 3.2 \\
\hline
\end{tabular}


Tabla 5. Distribución de parámetros productivos por clases diamétricas de roble negro (Colombobalanus excelsa) en el PNN Cueva los Guácharos y su zona de amortiguación, 2010

\begin{tabular}{lrrrrrrrrrrr}
\hline \multicolumn{1}{c}{ Parámetro } & $\mathbf{1 0 - 2 0}$ & $\mathbf{2 0 - 3 0}$ & $\mathbf{3 0 - 4 0}$ & $\mathbf{4 0 - 5 0}$ & $\mathbf{5 0 - 6 0}$ & $\mathbf{6 0 - 7 0}$ & $\mathbf{7 0 - 8 0}$ & $\mathbf{8 0 - 9 0}$ & $\mathbf{9 0 - 1 0 0}$ & \multicolumn{100}{c}{} & \multicolumn{1}{c}{ Total } \\
\hline Individuos ha $^{-1}$ & 92.66 & 61.66 & 34.33 & 19.68 & 20 & 15 & 11 & 4.33 & 10.66 & 12.34 & 281.66 \\
Área basal & 1.59 & 2.77 & 3.30 & 3.06 & 4.75 & 4.88 & 4.96 & 2.24 & 7.60 & 17.15 & 52.33 \\
Volumen & 14.94 & 30.82 & 40.95 & 39.54 & 71.99 & 68.92 & 70.96 & 33.62 & 108.52 & 281.35 & 761.65 \\
\hline
\end{tabular}

El modelo para estimar la altura de los árboles de C. excelsa en función del diámetro a la altura del pecho con el mejor ajuste fue el inverso de Harris, el cual presentó un coeficiente de correlación de 0.95 y un error estándar de 2.15 (Figura 1). Los otros seis modelos con sus respectivos coeficientes y errores se presentan en la tabla 6. Por otro lado, para la estimación del volumen, un modelo logístico resultó el de mejor ajuste, con un coeficiente de correlación de 0.988 y un error estándar de 0.623. La figura 2 muestra la función del modelo logístico y los modelos generados para la estimación de volumen de $C$. excelsa, se relacionan con sus respectivos estadísticos en la tabla 7.

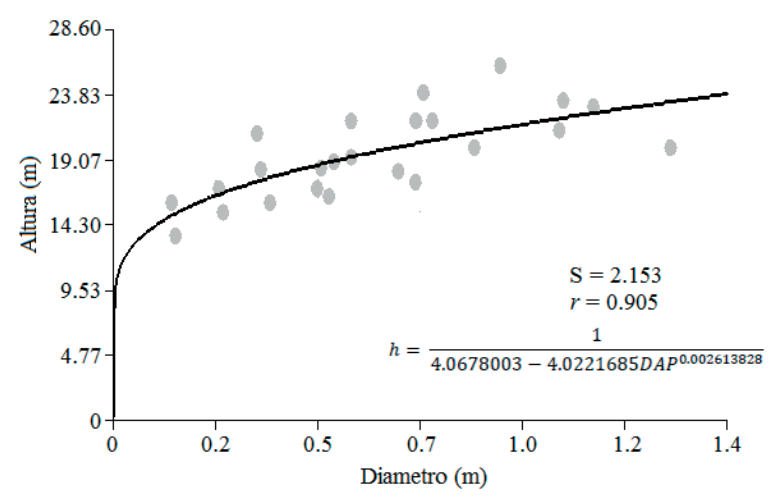

Figura 1. Modelo de Harris para estimación de la altura de roble negro (Colombobalanus excelsa) en función del diámetro a la altura del pecho. Ajustado con la información de 25 individuos

Tabla 6. Ecuaciones de altura para roble negro (Colombobalanus excelsa), en el PNN Cueva de los Guácharos. Ajustadas con información de 25 individuos, 2011

\begin{tabular}{lccc}
\hline \multicolumn{1}{c}{ Nombre } & Modelo & R & S \\
\hline Harris & $h=\frac{1}{4.0678003-4.0221685 D A P^{0.002613828}}$ & 0.95 & 2.2 \\
MMF & $h=\frac{(0.0081682432 * 1.72209322)+\left(59.4206117 D A P^{0.299099}\right)}{1.72209322+D A P^{0.2990991921708}}$ & & \\
Racional & $h=\frac{0.04867282+317.901082 D A P}{1+15.67175 D A P-1.9270127 D A P^{2}}$ & & 2.2 \\
Polinómica & $h=1.5285354+111.167732 D A P-263.35714 D A P^{2}$ \\
Exponencial & $\quad+270.59077 D A P^{3}-96.54472 D A P^{4}$ & 0.90 & 2.2 \\
Exponencial & $h=19.6843075051\left(1.06900515003-e^{-5.77547673277 D A P}\right)$ & 0.91 & 2.2 \\
\hline
\end{tabular}

Tabla 7. Ecuaciones de volumen para roble negro (Colombobalanus excelsa), en el PNN Cueva de los Guácharos. Ajustadas con información de 25 individuos, 2010

\begin{tabular}{lccc}
\hline \multicolumn{1}{c}{ Nombre } & Ecuación & R & S \\
\hline Logístico & Vol $=\frac{16.3963586558}{1+122.219883521 e^{-4.99811455720 D A P}}$ & 0.98 & 0.62 \\
MMF & Vol $=\frac{(0.239843 * 1.632655)+\left(1.63265483 D A P^{3.53812727916}\right)}{1.63265483+D A P^{3.53812727916}}$ & & \\
\hline
\end{tabular}




\begin{tabular}{lccc}
\hline \multicolumn{1}{c}{ Nombre } & Ecuación & R & S \\
\hline $\begin{array}{l}\text { Polinómica } \\
\text { de } 4^{\circ} \text { orden }\end{array}$ & Vol $=-0.07672955+3.51678 D A P-14.66696 D A P^{2}$ \\
$+34.74198 D A P^{3}-14.688361 D A P^{4}$ & 0.98 & 0.65 \\
Racional & $\mathrm{Vol}=\frac{-0.3463232+2.2265286 D A P}{1-1.30631778 D A P+0.5206932 D A P^{2}}$ & 0.98 & 0.66 \\
Función & $\mathrm{Vol}=13.231368+13.195000 \cos (1.476966 D A P+2.877088)$ & 0.98 & 0.67 \\
Sinusoidal & $\mathrm{Vol}=0.062586738-2.0158461 D A P+10.296868 D A P^{2}$ & 0.98 & 0.69 \\
Cuadrática & $\mathrm{Vol}=-2.19991045\left(1.3964634-e^{1.62123596967 D A P}\right)$ & 0.98 & 0.80 \\
Exponencial & & & \\
\hline
\end{tabular}

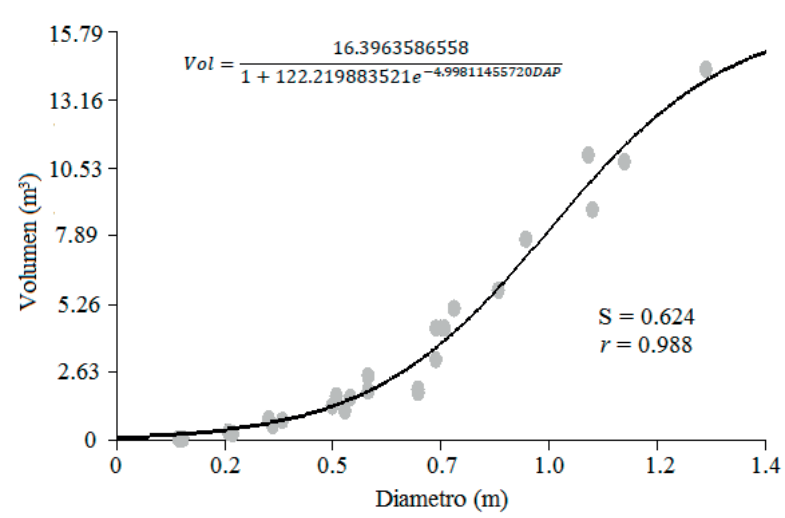

Figura 2. Modelo logístico para estimación del volumen de roble negro (Colombobalanus excelsa) relacionando el DAP

\section{DISCUSIÓN}

Con el presente estudio se logró evidenciar que las coberturas de roble negro $C$. excelsa en el área de distribución natural adyacente al PNN Cueva de los Guácharos se están reduciendo al igual que en otras zonas del país, donde esta especie comparte hábitat con el roble común $Q$. humboldtii. Esto se pudo corroborar con la existencia de fragmentos de bosque de roble negro rodeados por potreros y cultivos agrícolas en esta zona. Lo anterior plantea a estas zonas como estratégicas para establecer programas de conservación y estudiar los aspectos asociados a estas dos especies forestales (Palacio \& Fernández, 2006). Además, bajo esta situación se vienen generando serias alteraciones de los ciclos hidrológicos, así como del carbono y sobre la biodiversidad como ha sido identificado en otras latitudes del trópico (Armesto et al., 1992). Estas actividades antrópicas afectan de manera considerable a la especie llevándola a aumentar su grado de vulnerabilidad tanto en esta zona, como a nivel nacional; de igual forma, opaca los esfuerzos que se vienen adelantando en el paisaje anexo al PNN Cueva de los Guácharos, desde los cuales se reconoce la importancia de los bosques de roble negro para la conservación de la biodiversidad en el nivel del paisaje (Manchola \& Parra, 2006).

Otro aspecto por señalar sobre $C$. excelsa es que la especie siempre se había registrado por encima de los $1800 \mathrm{~m}$ de altitud; sin embargo, para este estudio se registraron rodales desde $10 \mathrm{~s} 1400 \mathrm{~m}$ de altitud, esto contrasta con lo mencionado por la Fundación Farallones (2009).

La obtención del factor de forma (FM) real o verdadero para esta especie genera una gran ventaja en cuanto a la precisión de la cuantificación de volumen de la especie C. excelsa, en sus diferentes clases diamétricas. Los métodos existentes de factor de forma falso y estándar de 0.7 para especies tropicales, que son calculados a partir de muestras de 10 a 15 árboles por especie, sobreestiman el volumen ya que las clases diamétricas que se encuentran entre 10 y $50 \mathrm{~cm}$ no alcanzan a tener un factor mórfico de 0.7 , mientras que para diámetros superiores este parámetro sería subestimado (CATIE, 1991; Prodán et al., 1997).

En relación con las existencias de área basal y volumen se puede observar que los bosques de roble negro $(C$. excelsa) de la zona presentan valores considerables en comparación con otros bosques de similares características, como los de Quercus humboldtii en la Cordillera Oriental y aun con los de Q. copeyensis Costa Rica, en donde el 
volumen por hectárea alcanza los $623 \mathrm{~m}^{3}$ (Blaser \& Camacho, 1991). El volumen para la zona de estudio fue de $761.65 \mathrm{~m}^{3} \mathrm{ha}^{-1}$, lo cual evidencia un alto potencial de estos bosques para almacenar carbono al igual que los bosques de $C$. excelsa registrados en Santander y Boyacá (AgudeloGuinand, 2009). El avance en el desarrollo de modelos de altura y de volumen en función del diámetro servirá de soporte para las mediciones posteriores que se realicen en estos bosques con fines de manejo, así como para la cuantificación de biomasa y carbono.

\section{CONCLUSIONES}

De acuerdo con la distribución espacial que presentó el roble negro en la zona de estudio, se puede afirmar que la mayor proporción de estos bosques se encuentra localizada sobre la zona amortiguadora del parque y que algunas de estas zonas en las cuales abunda este tipo de roble representan las áreas más grandes. Por otro lado, se pudo observar que las variables de estructura de los rodales evaluados en los diferentes sitios de muestreo presentaron valores homogéneos, lo que se pudo corroborar con errores de muestreo bajos, esto indica que el tamaño de las muestras es recomendado para futuros estudios encaminados al manejo y conservación de estos bosques. El diámetro presentó una relación directa y estrecha con la altura y el volumen en pie de los árboles, esto permitió construir varias ecuaciones para estimar de manera rápida y precisa estos atributos. Este hecho facilitará la realización de otros estudios orientados hacia el manejo y conservación de estos bosques, también ayudará a que estos sean incorporados en proyectos REDD y $\mathrm{REDD}^{+}$por el alto potencial que los mismos representan.

\section{AGRADECIMIENTOS}

Este trabajo fue desarrollado gracias a la colaboración de la Universidad del Cauca, a la asesoría de los profesores de la Facultad de Ciencias Agropecuarias y al apoyo logístico y técnico del PNN Cueva de los Guácharos, de las comunidades de San José de Riecitos, La Mensura y La Ilusión. Al coordinador del colegio San Adolfo don Federico Sterling Rincón y a las emisoras comunitarias San Adolfo 107 Digital de Palestina y la HJ doble K Pitalito.

\section{REFERENCIAS BIBLIOGRÁFICAS}

Agudelo-Guinand, M.I. (2009). Biomasa aérea y contenido de carbono en bosques de Quercus humboldtii y Colombobalanus excelsa: corredor de conservación de robles Guantivañ-La Rusia-Iguaque (SantanderBoyacá) (Tesis de pregrado, Administrador del medio ambiente). Santiago de Cali: Facultad de ciencias básicas, Universidad Autónoma de Occidente. 122 p.

Armesto, J., Smith-Ramírez, C., León, P., \& Arroyo, M. (1992). Biodiversidad y conservación del bosque templado en Chile. Ambiente y Desarrollo, 8, 19-24.

Blaser, J., \& Camacho, M. (1991). Estructura, composición y aspectos silviculturales de un bosque de (Quercus spp) del piso montano en Costa Rica, (Serie Técnica, informe técnico No. 185). Turrialba: Centro Agronómico Tropical de Investigación y Enseñanza (CATIE). 68 p.

Bustamante, R., \& Grez,A. (1995). Consecuencias ecológicas de la fragmentación de los bosques nativos. Ciencia y ambiente, 11(2), 58-63.

Cárdenas, D., \& Salinas, N. (eds.). (2006). Libro Rojo de Plantas de Colombia: Especies maderables amenazadas ( $1^{\circ}$ parte). Bogotá: Instituto Amazónico de Investigaciones Científicas (SINCHI).

CATIE [Centro Agronómico Tropical de Investigación y Enseñanza]. (1991). Manual práctico de mediciones de especies de árboles de uso múltiple. Turrialba: CATIE. $50-78$ p. 
Dávila, D. (2010). Cuantificación de volumen de la especie Colombalanus excelsa, en el Parque Nacional Natural Cueva de los Guácharos y su zona de amortiguación, Municipios de Palestina y Acevedo, Departamento del Huila (Tesis de pregrado, Ingenieria forestal). Popayán: Universidad del Cauca. 52 p.

Fundación Farallones. (2009). Fuerza viva de la Naturaleza, Roble Negro Colombobalanus excelsa. Cali, Colombia. Recuperado el 20 de diciembre de 2011 de http://www. fundacionfarallones.org/roblenegro.htm.

Galindo, R., Betancourt, J., \& Mendoza, H. (2006). Estructura y composición florística de los bosques de roble en dos Parque Nacionales Naturales en la Cordillera Oriental colombiana. En C. Solano \& N. Vargas (eds.). Memorias del I Simposio Internacional de Roble y Ecosistemas Asociados (pp. 95-100). Bogotá: Universidad Javeriana.

Hyams, D. (2010). CurvExpert Basic Release 1.4: Quick Start Guide. Recuperado de http:// docs.curveexpert.net/curveexpert/basic/_ static/CurveExpertBasic.pdf.

IAVH [Instituto Alexander von Humboldt]. (2002). Caracterización biológica del PNN Cueva de Los Guácharos. Bogotá: Instituto Alexander von Humboldt - Grupo GEMA. $50 \mathrm{p}$.

IBM. (2010). Guía breve de IBM SPSS Statistics 19. Recuperado el 20 de diciembre de2011 de http://www.unileon.es/ficheros/ servicios/informatica/spss/spanish/IBMSPSS_guia_breve.pdf.

Kattan, G.H. (2002). Fragmentación: patrones y mecanismos de extinción de especies. En M.R. Guariguata \& G.H. Kattan (eds.). Ecología y conservación de bosques neotropicales (pp. 561-590). Cartago: EULAC-GTZ.
Louman, B., Quirós, D., \& Nilsson, M. (1991). Silvicultura de bosques latifoliados húmedos con énfasis en América Central (Serie Técnica. Manual técnico No 46). Turrialba: CATIE. 125 p.

Manchola, H., \& Parra., C. (2006). Los robles como objeto de conservación del proyecto corredor biológico entre los PNN Puracé Cueva de los Guácharos. En C. Solano \& N. Vargas (eds.). Memorias del I Simposio Internacional de Roble y Ecosistemas Asociados (pp. 275). Bogotá: Universidad Javeriana.

Murcia, C. (1995). Edge effects in fragmented forest: implications for conservation. Tree, $10(2), 58-62$.

Orozco, L. (1991). Estudio ecológico y de estructura horizontal de seis comunidades boscosas en la cordillera de Talamanca (serie técnica, informe técnico No. 176). Turrialba: Centro Agronómico Tropical de Investigación y Enseñanza CATIE. 24 p.

Palacio, J., \& Fernández, J. (2006). Estado de la investigación en genética de la conservación de los robles (Fagácea) en Colombia. En C. Solano \& N. Vargas (eds.). Memorias del I Simposio Internacional de Roble y Ecosistemas Asociados (pp. 5772). Bogotá: Universidad Javeriana.

Prodán, M., Peters, R., Cox, F., \&. Real., P. (1997). Mensura Forestal. San José de Costarica: Instituto Interamericano de Cooperación para la Agricultura (IICA). $135 \mathrm{p}$.

Wadsworth, F. (1997). Forest production for tropical America. Washington: USDA, Forest Service, Agriculture Handbook. $320 \mathrm{p}$.

Williams, G. (1991). Los bordes de selvas y bosques. Ciencia y desarrollo, 17(97), 65-71. 